\title{
Assessment of Average Glandular Dose Received in Full-field Digital Mammography and Digital Breast Tomosynthesis
}

\author{
Napat Ritlumlert, M.Sc. ${ }^{1}$, Sutthirak Tangruangkiat, M.S. ${ }^{1}$, Monchai Phonlakrai, M.Sc. ${ }^{1}$ \\ Supannika Kawvised, Ph.D. ${ }^{1}$, Phornpailin Pairodsantikul, M.Sc. ${ }^{1}$, Sirachat Vidhyarkorn, M.D. ${ }^{2}$ \\ ${ }^{1}$ Faculty of Health Science Technology, HRH Princess Chulabhorn College of Medical Science, Chulabhorn Royal Academy, \\ Laksi, Bangkok 10210, Thailand. \\ 2Diagnostic Radiology, Chulabhorn Hospital, Chulabhorn Royal Academy, Laksi, Bangkok 10210, Thailand. \\ Received 2 September 2019 • Revised 2 January 2020 • Accepted 10 January 2020 • Published online 19 March 2020
}

\begin{abstract}
:
Objective: To assess the average glandular doses (AGD) from full-field digital mammography (FFDM) and digital breast tomosynthesis (DBT).
\end{abstract}

Material and Methods: Radiographic exposure parameters target/filter, tube voltage, and tube current were collected from 50 patients. Patient information including age, breast thickness, entrance surface air kerma (ESAK) and AGD from the monitor display were also recorded. The tube outputs (tube voltage and tube loadings) at the reference points in both FFDM and DBT modes were measured. The AGD was calculated from ESAK by using the correction factors following the Technical Report Series no. 457 protocol. For the DBT mode, the AGD was calculated and corrected for the $\mathrm{X}$-ray gantry rotation following the Dance et al. method.

Results: The radiation doses to breasts in terms of ESAK and AGD from FFDM were 4.97 \pm 2.29 and $1.36 \pm 0.48$ milligray (mGy) respectively. The third quartiles were $6.5 \mathrm{mGy}$ and $1.67 \mathrm{mGy}$, findings which were lower than the standard Dose Reference Levels reported by the International Atomic Energy Agency recommendation (AGD 3 mGy/view for standard breast thickness with grid). For the DBT mode, ESAK and AGD were $6.49 \pm 2.10 \mathrm{mGy}$ and $1.63 \pm 0.51 \mathrm{mGy}$. The third quartiles were $7.68 \mathrm{mGy}$ and $1.81 \mathrm{mGy}$ which were more than the FFDM mode by $23.0 \%$ and $17.0 \%$, respectively. Conclusion: This study found that the AGD received from the DBT mode was higher than from the FFDM mode. Patients who underwent combination modes of mammographic examination increasingly received AGD up to 1.74 mGy. However, the AGD in our institute was still lower than the standard AGD recommendations.

Keywords: average glandular dose, breast, digital breast tomosynthesis, digital full-field mammography, mammography

Contact: Napat Ritlumlert, M.Sc.

HRH Princess Chulabhorn College of Medical Science, Chulabhorn Royal Academy, Laksi, Bangkok 10210, Thailand.

E-mail: napat.rit@pccms.ac.th

(c) 2020 JHSMR. Hosting by Prince of Songkla University. All rights reserved.

This is an open access article under the CC BY-NC-ND license

(http://www.jhsmr.org/index.php/jhsmr/about/editorialPolicies\#openAccessPolicy).
J Health Sci Med Res 2020;38(2):115-123 doi: $10.31584 /$ jhsmr.2020730 www.jhsmr.org 


\section{Introduction}

Currently, breast cancer is the most common type of cancer in Thai women and second highest in terms of mortality rates. ${ }^{1}$ It can be found in teenagers but it is most common in women aged over 40. Early detection is an important strategy to prevent deaths from breast cancer. There are various methods of clinical breast examination such as palpation, ultrasound and screening mammogram. Mammogram is the most effective tool for detecting breast cancer at an early stage.

Full-field digital mammography (FFDM) is a type of breast imaging using solid-state detectors to directly convert $\mathrm{X}$-rays to electrical signals to produce digital $2 \mathrm{D}$ mammography. FFDM plays an important role in breast cancer screening and diagnosis because of low noise detection with higher detection efficiency. It also provides better soft tissue contrast and has the capability to detect early micro-calcification. Also, this system has a wide dynamic range and allows information to be stored and transferred in digital form. ${ }^{2}$ Additionally, digital breast tomosynthesis (DBT) is an imaging technique implemented in this modality that can decrease the effect of tissue overlapping. By using an X-ray gantry, the machine can be rotated and acquire images of a compressed breast from multiple angles. Subsequently, the obtained raw data can then be reconstructed into 3D breast images. As a result, this technique increases diagnostic capability. However, it also increases the radiation dose to the breast in the same time.

Measurement of average glandular dose (AGD) is very important but it is also very complicated as there are many factors which need to be considered in calculating an AGD in a digital mammography system. A study by Olgar et al. ${ }^{3}$ examined the AGD in digital mammography and found that the dose in the DBT mode was higher than in the FFDM mode by $34.0 \%$ in 52.7 millimeters $(\mathrm{mm})$ compression breast thickness (CBT) in craniocaudal view (CC view), and $56 \mathrm{~mm}$ in mediolateral oblique view (MLO view). They also reported that AGDs received from FFDM were 1.82 milligray (mGy) in the CC view and 1.94 mGy in the MLO view, while AGDs in the DBT mode were $2.53 \mathrm{mGy}$ in the CC view and 2.63 mGy in the MLO view. In addition, Svahn et al. ${ }^{4}$ reported that using stand-alone DBT operated at a low to slightly high dose resulted in an overall radiation dose $45.0 \%$ lower the FFDM. They proposed that by using only the DBT mode it would be possible to replace FFDM by reconstructing synthetic 2D images from 3D images, which would reduce AGD by half. Suleiman et al. ${ }^{5}$ collected dose reference levels (DRLs) from 1,537 studies, and stated that there are several methods to evaluate AGD received from different procedures ${ }^{6,7}$ as the comparison of DRLs from different procedures is very difficult and it is hard to get accurate values. Nguyen et al. $^{8}$ assessed the relationship between breast density and radiation dose by retrospectively screening mammography data, and found that breast thickness was the primary determinant of dose. They stated that compressed breast thickness was the major factor in received AGD, breast density was a minor factor and body mass index as well as patient's age had minimal impacts on dose levels, and suggested that decreasing breast compression thickness while performing mammography examinations was recommended to reduce radiation dose to the breast. However, this method causes patient pain and is uncomfortable.

As Asian woman have more dense breast tissue than Western women ${ }^{9}$, it might affect the accuracy of radiation dose evaluation in this group of patients. The assessment of the radiation dose from mammographic procedures is very important to ensure that the radiation dose received by any individual patient does not exceed the standard level while maintaining optimal image quality. Although a study of AGD in Thai women was performed by Anong $^{10}$, their investigation was performed on heterogeneous 
data acquired from different mammogram machines and DBT was not included.

The purpose of this study was to assess the AGD in both FFDM and DBT modes and compare them with DRLs in Thai women who underwent mammographic examination at Chulabhorn Hospital, Thailand.

\section{Material and Methods}

This was a retrospective study. Ethical approval was given by the Human Research Ethics Committee, Chulabhorn Research Institute. Fifty consecutive patients with age ranging from $35-70$ years who presented to the Department of Diagnostic Radiology of Chulabhorn Hospital and underwent digital mammographic examination were enrolled in the study.

The mammographic studies were performed using a Selenia Dimension, Hologic machine. The method described in the international atomic energy agency (IAEA) TRS 457 Protocol $^{11}$ was used to calculate the mean glandular FFDM doses. The incident air kerma for each patient's exposure was estimated from the measured X-ray tube output, measured with a Radcal ionizing chamber at the reference point for FFDM. The incident air kerma was measured from X-ray exposure, and conversion coefficients were used to calculate the AGD for each individual patient according to Equation 1:

$$
A G D=C_{D G 50 K i} C_{D g C G 50} s K_{i}
$$

Equation 1

Where $C_{D G 50 k i}$ and $C_{D g C G 50}$ are the conversion coefficients used for calculating AGD for breast of $50.0 \%$ glandularity and $0.1-100.0 \%$ glandularity at the central region of the breast, respectively, $S$ is the spectral correction and $K_{i}$ is the incident air kerma.

To perform breast dosimetry in DBT mode, the incident air kirma was measured in the $0^{\circ}$ projection and the $3 D$ breast dose $\left(D_{T}\right)$ of completed acquisition was calculated by Equation 2:

$$
D_{T}=K_{T} g C s T
$$

Equation 2

Where $K_{T}$ is incident air kerma, $g$ is the conversion factor for breast $50.0 \%$ against breast thickness and half value layer $(\mathrm{HVL}), c$ is the conversion factor for breasts with different glandularity, and $s$ is the conversion factor for different $\mathrm{X}$-ray spectra and T-factor for different scan ranges with the full-field detector geometry following Dance et al. $^{2}$

The exposure parameters of the combination modes used in all patients were collected, including average kilovoltage peak (kVp), mAs, target/filter, Anode, and CBT. antrance surface air kerma (ESAK) and AGD on the monitor screen were also recorded for verification. Each patient received 8 exposures in two modes. The first 4 exposures were performed in FFDM to produced images in the Right CC, Right MLO, Left CC and Left MLO views and the second 4 exposures were performed in the DBT mode. Patients with breast implants or who were imaged in special added positions were excluded from the study. All data were processed and analyzed using Stata/SE version 12 software (StataCorp LP, College Station, TX, United States).

\section{Results}

Table 1 shows the characteristics of the study women who underwent digital mammography at the Diagnostic Radiology Department in Chulabhorn Hospital. The patients were divided into two groups, screening (48.0\%) and diagnostic (52.0\%). The average patient age was $53.3 \pm 8.89$ years. The average compression breast thickness (CBT) was $53 \mathrm{~mm}$. The CBT and AGD were related with $p$-value $<0.001$ in which each $1 \mathrm{~mm}$ increase in breast thickness resulted in increased dose to the breast of 0.03 mGy in FFDM and 0.04 mGy in DBT.

Table 2 shows the parameters used in the FFDM (2D) and the DBT (3D) modes. The average kVp of DBT performed in the CC view was $31.36 \pm 2.07$, while it was 
$31.31 \pm 1.99$ in the MLO view. The $k V p$ used in the DBT mode was significantly higher than the $k V p$ used in the FFDM mode ( $p$-value<0.001). The $k V p$ differences in the two modes were 2.09 and $2.10 \mathrm{kVp}$, respectively. The mAs values of DBT in the CC and MLO views were 30.26 \pm 10.28 and $60.77 \pm 10.19$, respectively, which were significantly lower than in the FFDM mode of $115.77 \pm$ 40.64 in the CC view and $110.91 \pm 39.60$ in the MLO view and the mAs differences in the two modes were 55.51 and 50.14, respectively. The mean CBTs were slightly different in the two views. The mean CBT in the CC projection mode was $52.83 \pm 11.05 \mathrm{~mm}$ and in the MLO projection it was $53.15 \pm 11.20 \mathrm{~mm}$. The use of the Target/ filter parameter in the CC and MLO projections depended on the CBT. Tungsten target/rhodium (W/Rh) was used at CBT $<70 \mathrm{~mm}$ and tungsten target/silver filter (W/Ag) was used at CBT >70 $\mathrm{mm}$, while the tungsten target/aluminium filter target/filter was used in all CBTs for the DBT mode.

Table 1 Patient information

\begin{tabular}{|c|c|c|}
\hline Variable & Number & Percentage \\
\hline Age (years) mean \pm S.D. & \multicolumn{2}{|c|}{$53.3 \pm 8.89$} \\
\hline \multicolumn{3}{|l|}{ Group } \\
\hline Screening & 24 & 48.0 \\
\hline Diagnostic & 26 & 52.0 \\
\hline \multicolumn{3}{|c|}{ Compression breast thickness (mm) } \\
\hline $30-39$ & 5 & 10.0 \\
\hline $40-49$ & 9 & 18.0 \\
\hline $50-59$ & 24 & 48.0 \\
\hline $60-69$ & 9 & 18.0 \\
\hline$\geq 70$ & 3 & 6.0 \\
\hline
\end{tabular}

S.D.=standard deviation

Table 2 Parameters used in full field digital mammography (2D mode) vs digital breast tomosynthesis (3D mode)

\begin{tabular}{|c|c|c|c|c|c|c|}
\hline Mode & \multicolumn{3}{|c|}{ Projection CC } & \multicolumn{3}{|c|}{ Projection MLO } \\
\hline \multicolumn{7}{|c|}{$k V p$} \\
\hline & & Diff. kVp & $p$-value & & Diff. kVp & p-value \\
\hline & & (3D-2D) & & & $(3 D-2 D)$ & \\
\hline 2D mode & $29.27 \pm 1.56$ & \multirow{2}{*}{2.09} & \multirow{2}{*}{$<0.001$} & $29.21 \pm 1.67$ & \multirow{2}{*}{2.10} & \multirow{2}{*}{$<0.001$} \\
\hline 3D mode & $31.36 \pm 2.07$ & & & $31.31 \pm 1.99$ & & \\
\hline \multicolumn{7}{|c|}{ mAs } \\
\hline & & Diff. mAs & $p$-value & \multirow{2}{*}{\multicolumn{3}{|c|}{$\begin{array}{l}\text { Diff. mAs } \\
\text { (3D-2D) }\end{array}$}} \\
\hline & & $(3 D-2 D)$ & & & & \\
\hline 2D mode & $115.77 \pm 40.64$ & \multirow{2}{*}{-55.51} & \multirow{2}{*}{$<0.001$} & $110.91 \pm 39.60$ & \multirow{2}{*}{-50.14} & \multirow{2}{*}{$<0.001$} \\
\hline \multirow[t]{2}{*}{ 3D mode } & $30.26 \pm 10.28$ & & & $60.77 \pm 10.19$ & & \\
\hline & & & \multicolumn{2}{|c|}{ CBT $(\mathrm{mm})$} & & \\
\hline Mean (S.D.) & $52.83 \pm 11.05$ & & & $53.15 \pm 11.20$ & & \\
\hline Max & 81.00 & & & 74.00 & & \\
\hline \multirow[t]{2}{*}{ Min } & 28.00 & & & 24.00 & & \\
\hline & & & \multicolumn{2}{|c|}{ Target/filter } & & \\
\hline \multirow[t]{2}{*}{ 2D mode } & & $\mathrm{W} / \mathrm{Rh}$ & & \multicolumn{3}{|c|}{$\mathrm{W} / \mathrm{Rh}$} \\
\hline & & $\mathrm{W} / \mathrm{Ag}$ & & \multicolumn{3}{|c|}{$\mathrm{W} / \mathrm{Ag}$} \\
\hline 3D mode & & W/AI & & \multicolumn{3}{|c|}{ W/Al } \\
\hline
\end{tabular}

Diff=difference, $\mathrm{kVp}=\mathrm{kilovoltage}$ peak, $\mathrm{CC}=\mathrm{craniocaudal}, \mathrm{Cl}=\mathrm{confidence}$ interval, $\mathrm{MLO}=$ mediolateral oblique, $\mathrm{mAs}=\mathrm{milliampere}-\mathrm{seconds}$, $\mathrm{CBT}=$ compression breast thickness, $\mathrm{mm}=$ millimeters, $\mathrm{W} / \mathrm{Rh}=$ tungsten target/rhodium, W/Ag=tungsten target/silver filter, $\mathrm{W} / \mathrm{Al}=$ tungsten target/aluminium filter, S.D.=standard deviation 
The results of the radiation dose assessment are shown in Table 3. The average ESAKs were $4.97 \pm 2.29$ mGy in the FFDM mode and 6.49 \pm 2.10 mGy in the DBT mode. The AGDs were $1.34 \pm 0.52$ and $1.78 \pm 1.00$ mGy in the FFDM and DBT modes, respectively. The percent differences between calculated ESAK/AGD and ESAK AGD as shown on the workstation screens were $0.4 \%$ and $1.5 \%$ in the FFDM mode and $1.4 \%$ and $8.8 \%$ for the DBT mode, respectively. The third quartiles of calculated ESAK and AGD in FFDM were 6.58 and 1.67 mGy, respectively. In DBT mode, the third quartiles were 7.68 and $1.81 \mathrm{mGy}$, respectively, which were higher than in the FFDM mode. The calculated ESAK and AGD of the combo mode, calculated from summation of the FFDM and DBT modes, were $11.46 \pm 4.30$ and $1.49 \pm 0.93 \mathrm{mGy}$, respectively. The third quartiles of ESAK and AGD in the combo mode were 7.32 and 1.74 mGy, respectively, which were still lower than the results reported by $\mathrm{Anong}^{10}$ and the IAEA recommendations ${ }^{12}$ as shown in Table 4.

Table 3 Entrance surface air kerma and average glandular dose in full field digital mammography compared with digital breast tomosynthesis

\begin{tabular}{|c|c|c|c|c|c|c|}
\hline \multicolumn{7}{|c|}{ Full field digital mammography } \\
\hline & Monitor display & Calculations & $\%$ Difference & Monitor display & Calculations & \% Difference \\
\hline & ESAK (mGy) & ESAK (mGy) & & AGD (mGy) & AGD (mGy) & \\
\hline Mean & $4.99 \pm 2.46$ & $4.97 \pm 2.29$ & 0.4 & $1.34 \pm 0.52$ & $1.36 \pm 0.48$ & 1.5 \\
\hline Max & 13.7 & 12.2 & - & 3.2 & 3.8 & - \\
\hline Min & 1.4 & 1.5 & - & 0.53 & 0.5 & - \\
\hline $3^{\text {rd }}$ quartile & - & 6.58 & - & - & 1.67 & - \\
\hline \multicolumn{7}{|c|}{ Digital breast tomosynthesis } \\
\hline Mean & $6.40 \pm 2.20$ & $6.49 \pm 2.10$ & 1.4 & $1.78 \pm 1.00$ & $1.63 \pm 0.51$ & 8.8 \\
\hline Max & 14.0 & 13.9 & - & 14.0 & 3.7 & - \\
\hline Min & 2.5 & 2.7 & - & 0.9 & 0.8 & - \\
\hline $3^{\text {rd }}$ quartile & - & 7.68 & - & - & 1.81 & - \\
\hline
\end{tabular}

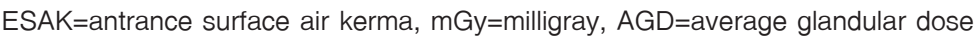

Table 4 Third quartile (75.0\% value) in entrance surface air kerma and average glandular dose compared to national dose reference levels and international atomic energy agency recommendations

\begin{tabular}{llclll}
\hline Mode & $\begin{array}{l}\text { Mean } \\
(\mathrm{mG})\end{array}$ & S.D. & $\begin{array}{l}3^{\text {rd }} \text { quartile } \\
(75 \% \text { value }) \\
(\mathrm{mGy})\end{array}$ & $\begin{array}{l}\text { Anong } \\
(\mathrm{mG})\end{array}$ & $\begin{array}{l}\text { IAEA } \\
\text { Recommendation } \\
(\mathrm{mGy})\end{array}$ \\
\hline 2D & \multicolumn{5}{c}{ Antrance surface air kerma } \\
3D & 4.97 & 2.29 & 6.58 & 12.9 & 11.0 \\
Combo (2D+3D) & 6.49 & 2.10 & 7.68 & - & - \\
\hline
\end{tabular}


Table 4 (continued)

\begin{tabular}{|c|c|c|c|c|c|}
\hline Mode & $\begin{array}{l}\text { Mean } \\
\text { (mGy) }\end{array}$ & S.D. & $\begin{array}{l}3^{\text {rd }} \text { quartile } \\
(75 \% \text { value }) \\
\text { (mGy) }\end{array}$ & $\begin{array}{l}\text { Anong } \\
\text { (mGy) }\end{array}$ & $\begin{array}{l}\text { IAEA } \\
\text { Recommendation } \\
\text { (mGy) }\end{array}$ \\
\hline \multicolumn{6}{|c|}{ Average glandular dose } \\
\hline $2 \mathrm{D}$ & 1.36 & 0.48 & 1.67 & 2.5 & 3.0 \\
\hline $3 \mathrm{D}$ & 1.63 & 0.51 & 1.81 & - & - \\
\hline Combo (2D+3D) & 1.49 & 0.93 & 1.74 & - & - \\
\hline
\end{tabular}

$m G y=$ milligrays, S.D.=standard deviation, IAEA=international atomic energy agency, Combo=combination 2D and 3D mode

\section{Discussion}

From our study, we identified many factors which can influence the average glandular dose (AGD) in FFDM.

The first factor is beast glandularity of patient. Typically, Asian women, including Thais, have higher breast densities with fibro glandular tissue over $50.0 \%$, compared with western women. As a result, it probably results to
AGD calculation in those who have the same compressed breast thickness. Our investigation found that increased AGDs corresponded to the increasing compressed breast thicknesses as shown in Figures 1 and 2, a finding also in other investigations. Also, the mean glandular doses, calculated from the ESAK, were related to the tube output measurements and correction factors.

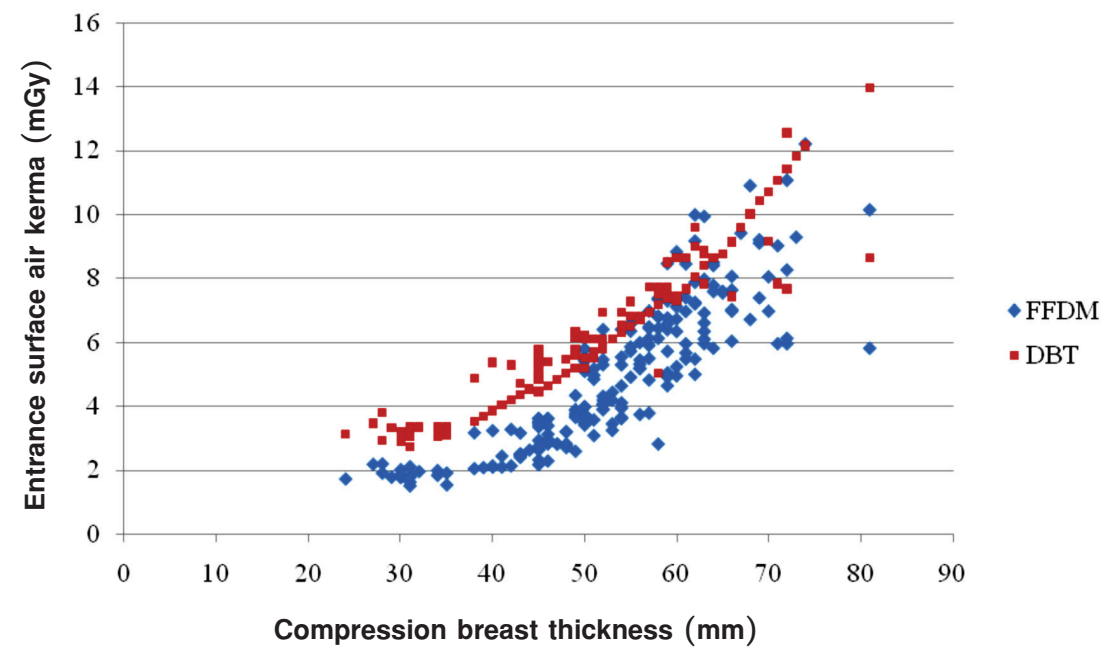

FFDM=full field digital mammography, DBT=digital breast tomosynthesis, mGy=milligrays, mm=millimeters

Figure 1 Entrance surface air kerma in full field digital mammography and digital breast tomosynthesis at the same compression breast thicknesses 


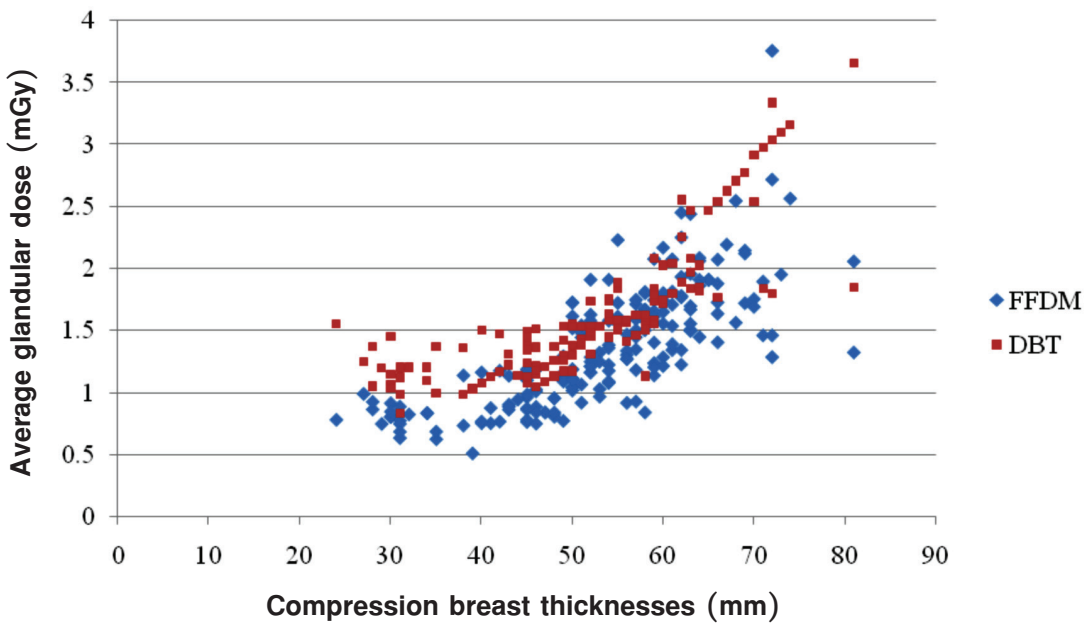

FFDM=full field digital mammography, DBT=digital breast tomosynthesis, $m G y=$ milligrays, $m m=$ millimeters

Figure 2 Average glandular dose in full field digital mammography and digital breast tomosynthesis at the same compression breast thicknesses

Secondly, using appropriate correction factors is also very important for accurate AGD calculations because selecting the correct conversion coefficient for the appropriate value of the HVL and breast thickness $\left(C_{D G 50, K i}\right)^{11}$ is required for accurate dose estimation because of different HVLs in each mammogram machine. In this study, we estimated the breast glandularity at $50.0 \%$ as a conversion coefficient to convert the mean glandular dose of the breast with $50.0 \%$ glandularity to the dose of breasts that had the same thickness but different glandularities $(\mathrm{g})\left(c_{D G g, D G 50}\right)^{11}$, acquired from different factors, such as target/filter combinations $(s)^{11}$ and scan ranges with the full-field detector geometry in DBT. From our calculations, we found that manually calculated AGDs and values on the monitor screen in the DBT mode had a large difference of $8.8 \%$, compared with FFDM at $1.5 \%$. This is probably because of using different correction factors in the system and in our method. However, these differences were within acceptable different values $(10.0 \%){ }^{13}$
Not only breast thickness, breast composition, and the correction factors affect breast dose level, but $k V p$, $\mathrm{mAs}$, and target/filter also result in the received radiation dose to the breast. ${ }^{14-16} \mathrm{mAs}$ and $\mathrm{kVp}$ factors are important factors related to AGD levels because the automatic filter mode and automatic exposure control (AEC) are used in digital mammography systems and the automatic adjustment of $\mathrm{mAs}$ and $\mathrm{kVp}$ by the system depends on the CBT. In this study, we found that a CBT of $70 \mathrm{~mm}$ was the cut-off thickness at which the system of our machine changed the type of target/filter, as a W/Rh target/filter was used when the CBT was lower than 70 $\mathrm{mm}$ but the machine switched to W/Ag when the CBT was over $70 \mathrm{~mm}$. Hence, these factors should be taken into account when AGDs are calculated for FFDM.

In our study, the mean CBT, calculated ESAK and AGD, and DBT were similar to the previous studies of Olgar et al. ${ }^{3}$ and Svahn et al. ${ }^{4}$ that the AGD of 3D mode was higher than the 2D mode. However, our study found lower doses compared with their studies. This probably 
resulted from using different breast glandular content estimations, as we used breast glandularity at $50.0 \%$. When we compared our results with the study of Anong ${ }^{10}$ conducted in Thai women, the AGD in the FFDM mode of our study was lower than their report as shown in Figure 3 and 4. These different results might have been the result of using different target material and systems suggested.

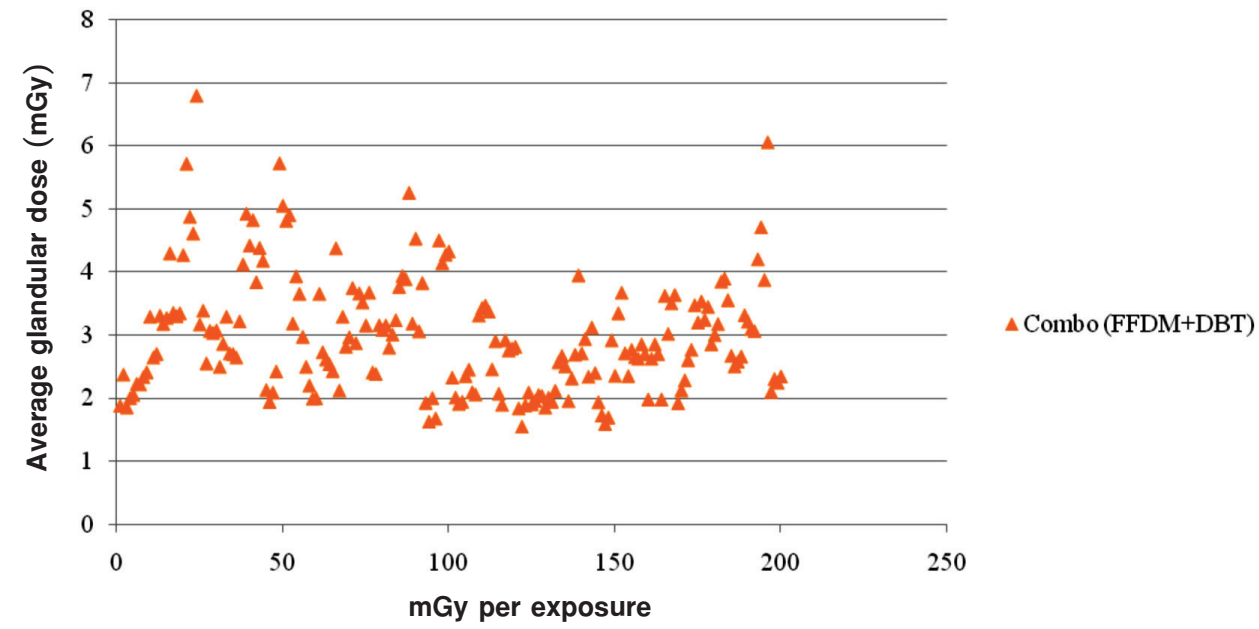

mGy=milligreys, FFDM=full field digital mammography, DBT=digital breast tomosynthesis, Combo=combination mode

Figure 3 Average glandular dose in combination mode (full field digital mammography plus digital breast tomosynthesis)

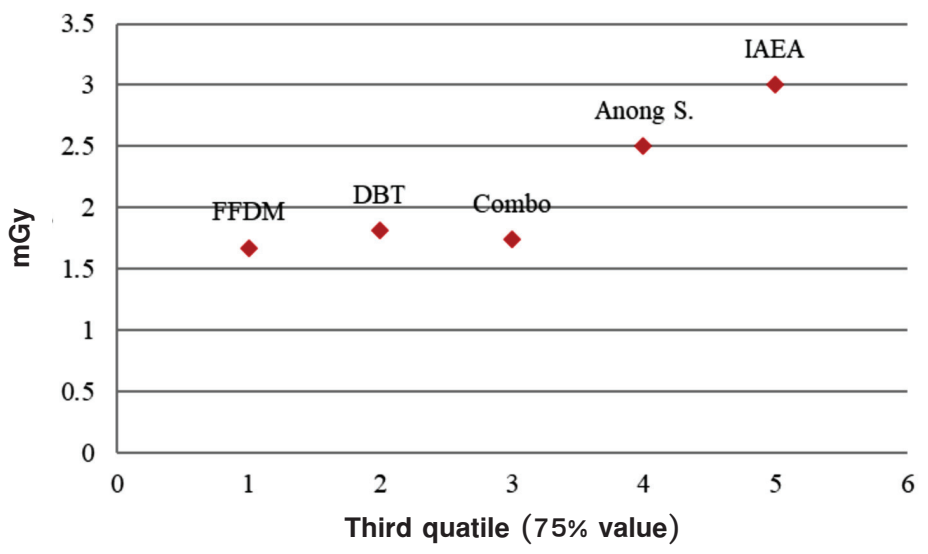

mGy=milligreys, FFDM=full field digital mammography, $\mathrm{DBT}=$ digital breast tomosynthesis, Combo=combination mode, IAEA=international atomic energy agency

Figure 4 Third quartile $(75.0 \%$ value) compared with dose reference levels 


\section{Conclusions}

Our study found that the AGD received in the DBT mode was higher than in FFDM. Patients who underwent a combo mammographic examination (FFDM plus DBT) received a higher mean glandular dose up to $1.74 \mathrm{mGy}$ in comparison with single mode investigations, but overall the mean glandular dose in our institute was lower than the standard DRLs.

\section{Acknowledgement}

The authors would like to thank the staffs of the Radiology Department, Chulabhorn Hospital and the $\mathrm{HRH}$ Princess College of Medical Sciences. Special thanks should be given to Kananan Utitsarn (Ph.D.), Medical Physicist, Lopburi Cancer Hospital for her guidance and language proofreading.

\section{Funding sources}

This study was supported by the HRH Princess Chulabhorn College of Medical Science, Chulabhorn Royal Academy.

\section{Conflict of interest}

The authors declare that there are no conflicts of interest.

\section{References}

1. Thailand National Cancer Institute, Department of Medical Services, Ministry of Public Health. Breast cancer screening in Thailand recommendations. Bangkok: Kosit Press; 2014.

2. Dance DR, Young KC, van Engen RE. Estimation of mean glandular dose for breast tomosynthesis. Phys Med Bio 2011: 453-71.

3. Olgar T, Kahn T, Gosch D. Average glandular dose in digital mammography and breast tomosynthesis. Rofo 2012;184: 911-8.

4. Svahn TM, Houssami N, Sechopoulos I, Mattsson S. Review of radiation dose estimates in digital breast tomosynthesis relative to those in two-view full-field digital mammography. Breast 2015;24:93-9.

5. Suleiman ME, Brennan PC, McEntee MF. Diagnostic reference levels in digital mammography: a systematic review. Radiat Prot Dosimetry 2015;167:608-19.

6. Sechopoulos I, Sabol JM, Berglund J, Bolch WE, Brateman L, Christodoulou E, et al. Breast tomosynthesis radiation dosimetry: task group 223 report. Med Phys 2014;41:091501.

7. Dance DR, Sechopoulos I. Dosimetry in x-ray-based breast imaging. Phys Med Biol 2016;61:R271-304.

8. Nguyen JV, Williams MB, Patrie JT, Harvey JA. Do women with dense breasts have higher radiation dose during screening mammography? Breast J 2018;24:35-40.

9. Greeshma AA, Ellen D, Yoon-Jin K, Priyamka H, Christopher $\mathrm{PH}$, Carl JD, loannis S. Can breast compression be reduced in digital mammography and breast tomosynthesis?. AJR Am J Roengenol 2017;209. doi: 10.2214/AJR.16.17615.

10. Anong S. Survey of mean glandular dose in mammography practically hospitals in the central region of Thailand. Med Sci 2012;58:33-8.

11. International Atomic Energy Agency. Technical Reports Series no. 457: Dosimetry in diagnostic radiology: an international code of practice. Vienna: Publishing Section IAEA; 2007.

12. Dance DR, Young KC, van Engen RE. Further factors for the estimation of mean glandular dose using the United Kingdom, European and IAEA breast dosimetry protocols. PhyMed Biol 2009:4361-72.

13. European Guidelines. European protocol for the quality control of the physical and technical aspects of mammography screening. Nijmegen: EUREF Office; 2013.

14. Fischer U, Hermann KP, Baum F. Digital mammography: current state and future aspects. Eur Radiol 2006;6:38-44.

15. Sechopoulos I, D’Orsi CJ. Glandular radiation dose in tomosynthesis of the breast using tungsten targets. J Appl Clin Med Phys 2008;9:2887.

16. Sung US, Jung MC, Min SB, Su HL, Nariya C, Mninae S, Won HK, Woo KM. Comparative evaluation of average glandular dose and breast cancer detection between singleview digital breast tomosynthesis (DBT) plus singleview mammography (DM) and two-view DM: correlation with breast thickness and density. Eur Radiol 2015;25:1-8. 